ISAHP 1999, Kobe, Japan, August 12-14, 1999

\title{
RECENT THORETICAL DEVELOPMENT OF AHP AND ANP IN JAPAN
}

\author{
Iwaro Takahashi \\ Department of Mathematical Engineering, \\ College of Industrial Technology, Nihon University \\ 1-2-1 Izumi-cho, Narashino, Chiba 275-8575, Japan
}

\begin{abstract}
Since T. Saaty founded AHP and ANP, these have been developed by many research workers. Recently especially Japanese workers are making remarkable theoretical works in this field. Here I would like to introduce the essences of them. Of course there must be many other excellent works I have not opportunity to know. So I am afraid this lecture is not overall report but rather biased one about this field.
\end{abstract}

\section{The mathematical foundation of eigen vector method}

T. Saaty[Saaty,1980] proposed such principle that the principal eigen vector of a comparison matrix $\boldsymbol{A}=\left[a_{i j}\right]$ are reasonable evaluating weights of the given objects. This intuitive insight by Saaty to invent the eigen vector method is certainly excellent. This method has been used in almost all application fields of AHP and made many successful results.

There is the explanation that if $a_{i j}=w_{i} / w_{j}$ then the principal eigen vector of $\boldsymbol{A}$ is $\boldsymbol{w}=\left[w_{1} \cdots w_{n}\right]^{T}$ so even if $a_{i j}$ is not exactiy to $w_{i} / w_{j}$, the principal eigen vector of $\boldsymbol{A}$ must be near to $\boldsymbol{w}$. But this is rather week as the theoretical foundation of eigen vector method.

For a long time I have been thinking over this problem and believing that there should be some fundamental principle to produce Saaty's eigen vector method. And at last K. Sekitani \& N. Yamaki[Sekitani,1999] found that it is Frobenius mini-max theorem that gives this principle.

His idea is as follows; $w_{i}$ is the self-evaluation of object $i$ and $a_{i j} w_{j}$ is the evaluation of $i$ from view of $j$, so the average of external evaluation of $i$ is

$$
\vec{w}_{i}=\sum_{j \neq i} a_{i j} w_{j} /(n-1)
$$

Therefor $w_{i}(i=1 \sim n)$ minimizing overall discrepancies between $w_{i}$ and $\bar{w}_{i}(i=1 \sim n)$ must be desirable estimation of weights of objects.

This idea is represented by the following mathematical language;

$$
\min _{w}\left\{\max _{i}\left(\frac{\sum_{j \neq i} a_{i j} w_{j} /(n-1)}{w_{i}}\right)\right\}
$$

The following Frobenius mini-max theorem just meets to get a solution of formula (1.2).

[Theorem 1] Frobenius mini-max theorem; For a non-negative $n \times n$ matrix $\boldsymbol{B}=\left[b_{i j}\right]$ and any positive vector $\boldsymbol{w}$ we have

$$
\min _{i}\left(\frac{\sum_{j=1}^{n} b_{i j} w_{j}}{w_{i}}\right) \leq \lambda_{\max } \leq \max _{i}\left(\frac{\sum_{j=1}^{n} b_{i j} w_{j}}{w_{i}}\right)
$$


( where $\lambda_{\max }$ is the principal (maximal) eigen vector of $\boldsymbol{B}$ ). And if $\boldsymbol{B}$ is irreducible we have

$$
\max _{w>0} \min _{i}\left(\frac{\sum_{j=1}^{n} b_{i j} w_{j}}{w_{i}}\right)=\lambda_{\max }=\min _{w>0} \max _{i}\left(\frac{\sum_{j=1}^{n} b_{i j} w_{j}}{w_{i}}\right)
$$

And the principal eigen vector $u$ of $B$ attains $\max$ or $\min$ in (1.4).

So taking $\widehat{A}=(A-I) /(n-1)$ as $B$, the right hand side of (1.4) teaches us that the solution of (1.2) is the principal eigen vector of $\widehat{A}$, but the eigen vector of $\widehat{A}$ coincides with that of $A$. (Because if $\boldsymbol{u}$ is the principal eigen vector of $\boldsymbol{A}$ then

$$
\widehat{A} u=\frac{1}{n-1}(A-I) u=\frac{\lambda_{\max }-1}{n-1} u
$$

so $u$ is also the principal eigen vector of $\widehat{A}$ and vice versa.)

Sekitani's theorem states that the weights $w_{i}(i=1 \sim n)$ minimizing the maximal ratio of the average $\bar{w}_{i}$ of external evaluations and the self evaluation $w_{i}$ is the principal eigen vector of comparison matrix $\boldsymbol{A}$.

This gives not only the foundation of Saaty's eigen vector method but also various solving methods of variations of AHP or ANP, further this make us free from the conventional restriction that the comparison matrix must be reciprocal.

Typical variation of AHP is the incomplete information case, that is, some elements of the comparison matrix $\boldsymbol{A}=\left[a_{i j}\right]$ are missing. For such problems Harker's method is well known. But I explain this through the following simple example.

\section{Example 1}

$$
A=\left[\begin{array}{cccc}
1 & 2 & 0.5 & 2 \\
0.5 & 1 & 2 & () \\
2 & 0.5 & 1 & 0 \\
0.5 & 0 & 0 & 1
\end{array}\right]
$$

(A missing element is shown by ())

$$
\begin{array}{cll}
\text { self evaluation } & & \text { average of external evaluations } \\
w_{1} & - & \left(2 w_{2}+0.5 w_{3}+2 w_{4}\right) / 3 \\
w_{2} & - & \left(0.5 w_{1}+2 w_{3}\right) / 2 \\
w_{3} & - & \left(2 w_{1}+0.5 w_{2}\right) / 2 \\
w_{4} & - & 0.5 w_{1}
\end{array}
$$

Note that in the complete case each object has the some number $(n-1)$ of the external evaluations, but in the incomplete case it differs depending on objects. So for this problem we have $\widehat{A}$ as

$$
\widehat{A}=\left[\begin{array}{cccc}
0 & 2 / 3 & 0.5 / 3 & 2 / 3 \\
0.5 / 2 & 0 & 2 / 2 & 0 \\
2 / 2 & 0.5 / 2 & 0 & 0 \\
0.5 & 0 & 0 & 0
\end{array}\right]
$$

And the principal eigen vector $\boldsymbol{w}$ of $\widehat{\boldsymbol{A}}$ is the desired weights of objects.

$$
w^{T}=\left[\begin{array}{llll}
0.278 \cdot 0.308 & 0.298 & 0.117
\end{array}\right]
$$


By Harker's method we have the solution of the above problem as the principal eigen vector of

$$
A^{\prime}=\left[\begin{array}{cccc}
1 & 2 & 0.5 & 2 \\
0.5 & 2 & 2 & 0 \\
2 & 0.5 & 2 & 0 \\
0.5 & 0 & 0 & 3
\end{array}\right]
$$

It is

$$
w^{\prime T}=\left[\begin{array}{llll}
0.286 & 0.310 & 0.302 & 0.102
\end{array}\right]
$$

which is near to but somewhat different from $w^{T}$. How different is explained in [Sekitani,ISAHP99].

Here we note that Sekitani's principle is to take the "average" of external evaluations to compare with the self-evaluation, but we can also take "total" instead of "average". By total-principle we have the following formula instead of (1.2).

$$
\min _{w} \max _{i}\left(\sum_{j \neq i} a_{i j} w_{j} / w_{i}\right)
$$

And the solution of (1.5) is the principal eigen vector of $A-I$.

\section{Example 2}

There are 4 members $1,2,3$ and 4 contending in a tournament contest of chess game. the result - was that like Fig. 1 ( 1 defeats 2 and 3 , and 3 defeats 4 ).

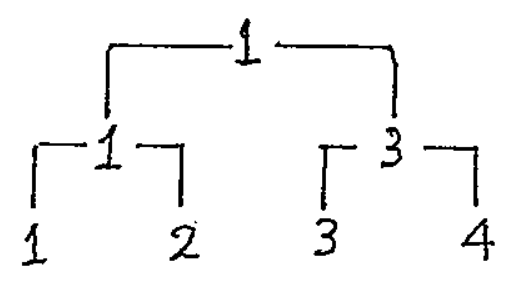

Fig. 1.

Let $a_{i j}=\theta$ and $a_{j i}=1 / \theta(\theta>1)$ if $i$ defeats $j$ and otherwise $a_{i j}=0$, then the comparison matrix is

$$
A=\left[\begin{array}{cccc}
1 & \theta & \theta & 0 \\
1 / \theta & 1 & 0 & 0 \\
1 / \theta & 0 & 1 & \theta \\
0 & 0 & 1 / \theta & 1
\end{array}\right]
$$

For this problem "total" principle is appropriate, so we take the principal eigen vector

$$
w^{T}=\left[\begin{array}{llll}
0.509 & 0.157 & 0.255 & 0.079
\end{array}\right]\left(\lambda_{\max }=1.618\right)
$$

of $A-I$ as the evaluations of members $(\theta=2)$.

But by Harker's method we have

$$
w^{T}=\left[\begin{array}{llll}
0.444 & 0.222 & 0.222 & 0.111
\end{array}\right]
$$

which coincides with the solution obtained by "average" principle[Sekitani,ISAHP99]. 


\section{The unifying method to solve AHP and ANP}

The analysis of ANP is based on the Saaty's so-called supermatrix[Saaty,1996]. Here I would like to explain what the supermatrix is through a simple example.

\section{Example 3}

In U.S.A there are three big fast-food companies, McDonald's(M), Burger King(B) and Wendy's(W). Assume that they are evaluated by two criteria advertisement(A) and service(S) like Table 1, and each of $M, B$ and $W$ has its management policy with weights for $A$ and $S$ like Table 2.

Table 1.

\begin{tabular}{|c|cc|}
\hline & $\mathrm{A}$ & $\mathrm{S}$ \\
\hline $\mathrm{M}$ & $u_{11}$ & $u_{12}$ \\
$\mathrm{~B}$ & $u_{21}$ & $u_{22}$ \\
$\mathrm{~W}$ & $u_{31}$ & $u_{32}$ \\
\hline
\end{tabular}

Table 2.

\begin{tabular}{|c|ccc|}
\hline & $\mathrm{M}$ & $\mathrm{B}$ & $\mathrm{W}$ \\
\hline $\mathrm{A}$ & $w_{11}$ & $w_{12}$ & $w_{13}$ \\
$\mathrm{~S}$ & $w_{21}$ & $w_{22}$ & $w_{23}$ \\
\hline
\end{tabular}

In a word $(A, S)$ evaluate $(M, B, W)$ and at the same time $(M, B, W)$ evaluate $(A, S)$. The evaluating matrix of $(\mathrm{M}, \mathrm{B}, \mathrm{W})$ by $(\mathrm{A}, \mathrm{S})$ is

$$
\boldsymbol{U}=\left[\begin{array}{ll}
u_{11} & u_{12} \\
u_{21} & u_{22} \\
u_{31} & u_{32}
\end{array}\right]
$$

and that of $(A, S)$ by $(M, B, W)$ is

$$
W=\left[\begin{array}{lll}
w_{11} & w_{12} & w_{13} \\
w_{21} & w_{22} & w_{23}
\end{array}\right]
$$

where standardizing conditions

$$
\sum_{i=1}^{3} u_{i j}=1(j=1,2), \sum_{i=1}^{2} w_{i j}=1(j=1 \sim 3)
$$

are assumed.

Saaty's supermatrix for this ANP is

$$
S=\left[\begin{array}{cc}
0 & W \\
U & 0
\end{array}\right]
$$

Because of (2.3) $S$ is a stochastic matrix. And analyses of ANP are always based on stochastic-ness of supermatrices.

Saaty[Saaty, 1996] proposed the limiting process $\lim _{t \rightarrow \infty} S^{t}$ to analyze ANP. Precisely speaking he proved the following theorem. 
[Theorem 2] If $S$ is irreducible and primitive $\lim _{t \rightarrow \infty} S^{t}$ converges to $S^{\infty}$ whose column vectors coincide with common vector $x$, that is,

$$
\lim _{t \rightarrow \infty} S^{t}=S^{\infty}=\left[\begin{array}{llll}
x & x & \cdots & x
\end{array}\right]
$$

and each element of $x$ is the required evaluating weight of the object.

Here irreducibility and primitivity of a matrix are important concepts. There can be well-explained graph theoretically. Considering an $\operatorname{arc}(i, j)$ if and only if $i$-th column element of matrix $M$ is non-zero, we have the directed graph corresponding to $M . M$ is irreducible if and only if its graph is strongly connected, and (non-negative) $M$ is primitive if and only if the greatest common divisor $d$ of lengths of all cycles in its graph is 1 .

It is easily found that $d$ of $S$ in (2.4) is 2, so $S$ in (2.4) is not primitive. For general case of $d \neq 1$, we have only to take $S^{d}$ instead of $S$, that is,

$$
\lim _{t \rightarrow \infty}\left(S^{d}\right)^{t}=\left[\begin{array}{llll}
x & x & \cdots & x
\end{array}\right]
$$

and $\boldsymbol{x}$ is the desired weight vector of objects.

Note that the above analyses are based on the stochastic-ness of $S$. And we can easily find[Sekitani] that $x$ in (2.5) or (2.6) coincides with the solution $x$ of

$$
S x=x
$$

The maximal (principal) eigen value of $S$ is 1 so, the solution $x$ of $(2.7)$ can be considered as the principal eigen vector of $S$.

From this fact even if $S$ is not stochastic, the principal eigen vector of $S$, that is the solution $u$ of

$$
S u=\lambda_{\max } u
$$

must be the desired evaluated weight vector of objects in ANP. But this is exactly the solution of Sekitani's formula (1.5) by taking $S$ as $\boldsymbol{A}$ in (1.5). And the following famous theorem of Frobenius gives the uniqueness and positivity of the solution of $(2.8)$.

[Theorem 3] The principal eigen vector of a non-negative irreducible matrix is unique and positive vector.

So Sekitani's principle is directiy applicable to ANP, and further by this we are free from the restriction of stochastic-ness of supermatrices. In a word in both AHP and ANP we can solve the problem by finding the principal eigen vector of the comparison matrix $A$ and the supermatrix $S$ respectively.

Generally the supermatrix $S$ in ANP is considered to be in the higher level than the comparisons matrix $\boldsymbol{A}$ in AHP. The word "super" itself reveals this fact. The elements of $\boldsymbol{A}$ are given by direct paired comparisons, but the elements of $S$ are given through several steps of calculations.

But mathematically they have the same role which generates the synthesized evaluations of objects. And synthesizing procedure is very simple. It is the calculation of the principal eigen vector of $A$ or $S$. 


\section{General solving method of ANP}

In section $2 \mathrm{I}$ explained that problems of ANP are solved by calculating the principal eigen vector of supermatrix $S$, and that this method need not the stochastic-ness of $S$. But as shown in section 2, the (non-negative) irreducibility of $S$ ( the strongly connectivity of its graph) is our basic condition. Saaty treats reducible (non-irreducible) supermatrices, but I think it ends in rather unsatisfactory results.

But very recently Sekitani \& Takahashi[Sekitani] had an idea to solve general (not necessarily irreducible) supermatrix $S$. Here I briefly introduce their idea.

Now the restriction imposed on $S$ becomes only non-negativity, but of course its graph must be connected, otherwise the problem is meaningless. We often encounter the case where the graph of $\boldsymbol{S}$ is connected but not strongly connected. Note that diagonal elements of $S$ are always zeros, that is, its graph has not self-loop.

\section{Example 4}

Consider again Example 3 (in section 2). Assume that general evaluations of $\boldsymbol{A}$ and $\boldsymbol{S}$ in fast food business word are $v_{1}$ and $v_{2}$ respectively and let $\boldsymbol{v}^{T}=\left[v_{1} v_{2}\right]$; then the supermatrix is

$$
S=\left[\begin{array}{ccc}
0 & 0 & 0 \\
v & 0 & W \\
0 & U & 0
\end{array}\right]
$$

and the corresponding graph is that in Fig. 2. This is clearly connected but not strongly connected.

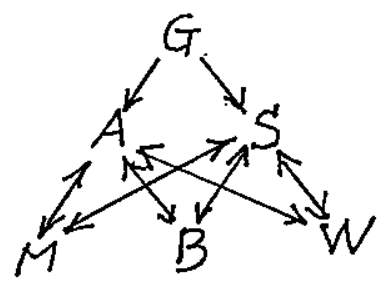

Fig. 2.

A connected graph is always decomposed to several strongly connected components (clusters) and each cluster has such order that an earlier ordered cluster has no input from lower ordered clusters. For example the graph in Fig. 3 has 4 clusters. And the corresponding super matrix is

$$
S=\left[\begin{array}{cccc}
A_{1} & 0 & 0 & 0 \\
B_{21} & A_{2} & 0 & 0 \\
B_{31} & 0 & A_{3} & 0 \\
0 & B_{42} & B_{43} & A_{4}
\end{array}\right]
$$

where $A_{i}$ is the evaluation matrix within cluster $i$, and $B_{i j}$ is the evaluation matrix of cluster $i$ by cluster $j$. And $A_{i}$ is irreducible unless $A_{i}$ is a $1 \times 1$ matrix, that is, a scalar. Through this example I explain the general solving algorithm.

Firstly the following standardizing procedure is necessary; for each $i$ (except the case $A_{i}$ is a scalar) calculate the principal eigen value $\lambda_{i}$ of $A_{i}$ and let

$$
\bar{A}_{i}=A_{i} / \lambda_{i}, \bar{B}_{\dot{k} i}=B_{k i} / \lambda_{i}(k=i+1, \cdots)
$$




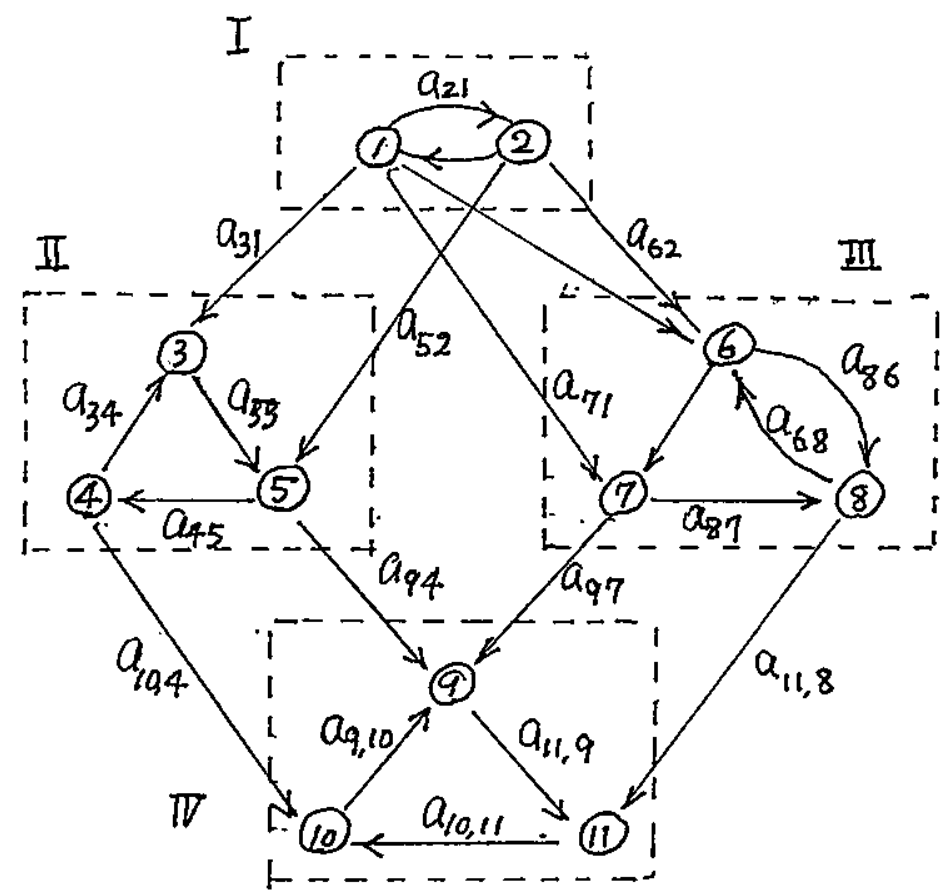

Fig. 3.

Then every principal eigen value of $\bar{A}_{i}$ is 1 (If $A_{i}$ is a scalar, it is always 0 , then assume $\bar{A}_{i}=0$ ).

This standardizing procedure not only plays the role to make evaluation levels of clusters even, but also make the following algorithm reasonable.

The solving algorithm based on the average-principle is as follows;

cluster I : $\bar{A}_{1}$ is irreducible, so the method of section 2 can be applied to have solution $u_{1}$ which is the evaluation weight vector of objects in cluster I, that is, $u_{1}$ is the principal eigen vector of $\bar{A}_{1}$. Here we assume that $u_{k}(k=1,2, \cdots)$ is always standardized so that the sum of elements is 1 . (If $\bar{A}_{1}=\left[a_{11}\right]$ is a scalar then $a_{11}=0$ (diagonal element is always zero), and we have $u_{1}=u_{11}=1$ ).

cluster II : Denote the number of non-zero elements in $i$-th row of [ $\bar{B}_{21} \bar{A}_{2}$ ] by $n_{2 i}$. And let $\left[\begin{array}{ll}\widehat{B}_{21} & \widehat{A}_{2}\end{array}\right]$ be the matrix whose $i-t h$ row is divided by $n_{2 i}$, and further $\widehat{b}_{2}=\widehat{B}_{21} u_{1}$. Then let the principal eigen vector of

be

$$
\left[\begin{array}{cc}
1 & 0 \\
\widehat{b}_{2} & \widehat{A}_{2}
\end{array}\right]
$$

$$
\left[\begin{array}{c}
1 \\
u_{2}
\end{array}\right]
$$

and $u_{2}$ is the evaluation weight vector of objects in cluster II.

cluster III : is solved by the same way as cluster II.

cluster IV : Let $\widehat{b}_{4}=\widehat{B}_{42} u_{2}+\widehat{B}_{43} u_{3}$, and let the principal eigen vector

$$
\left[\begin{array}{c}
1 \\
u_{4}
\end{array}\right]
$$


of (3.5) be then $u_{4}$ is the solution of cluster IV $\left(\widehat{A}_{4}, \widehat{B}_{43}, \cdots\right.$ are the same meaning as $\widehat{A}_{2}$ and $\widehat{B}_{21}$ ).

$$
\left[\begin{array}{cc}
1 & 0 \\
\widehat{b}_{4} & \widehat{A}_{4}
\end{array}\right]
$$

Note that the matrix of type(3.4) (or (3.5)) is not irreducible so generally we have no evidence that the principal eigen vector of this is a positive vector. But the principal eigen value of $\overline{\boldsymbol{A}}_{k}$ is always 1 , so that of $\widehat{A}_{k}$ is smaller than 1 so the principal eigen value of the matrix of type(3.4) is always 1 because of value 1 of $(1,1)$ element; which shows that the principal eigen vector (3.4) is the solution of

$$
\widehat{b}_{k}+\widehat{A}_{k} u_{k}=u_{k}
$$

Formula (3.6) shows that the self-evaluation of each object is equal to the average of the external evaluations. Further more the following theorem gives the basic foundation of the above algorithm.

[Theorem 4] For the principal eigen vector

$$
\left[\begin{array}{c}
1 \\
u_{k}
\end{array}\right]
$$

of

$$
\left[\begin{array}{cc}
1 & 0 \\
\widehat{b}_{k} & \widehat{A}_{k}
\end{array}\right]
$$

$u_{k}$ is a solution of the problem

$$
\min _{u_{k}>0} \max _{i}\left\{\max \left(\frac{\widehat{b}_{i}(k)+\sum_{j} \widehat{a}_{i j}(k) u_{j}(k)}{u_{i}(k)}, \frac{u_{i}(k)}{\widehat{b}_{i}(k)+\sum_{j} \widehat{a}_{i j}(k) u_{j}(k)}\right)\right\}
$$

where $\widehat{a}_{i j}(k)$ is the $(i, j)$ element of $\widehat{A}_{k}$ and $\widehat{b}_{i}(k)\left(u_{i}(k)\right)$ is $i$-th element of $\widehat{b}_{k}\left(u_{k}\right) \mathbf{n}$.

Formula (3.7) is almost similar to our fundamental criteria (1.2), and gives the evidence of positivity of $u_{k}$.

\section{Dominant alternatives and the concurrent convergent method}

Elements of the comparison matrix $\boldsymbol{A}$ in AHP or the supermatrix $S$ in ANP can be considered to be relative evaluation values. The analysis of AHP or ANP is to be estimate the absolute evaluation values of objects by the given values of $A$ or $S$. Speaking graph theoretically, we are to estimate the unknown values of nodes by, the given values of arcs.

But the values of the elements of $\boldsymbol{A}$ or $S$ themselves are often very unstable or erroneous. It is often said that the evaluation of alternatives by each criterion is rather stable but the evaluation of weights of criteria in ANP is very unstable.

Kinoshita \& Nakanishi[Kinoshita,1999] proposed very unique ideas "the dominant alternatives" and "the concurrent convergent". these can be used to correct the unstable or erroneous values of evaluations. In the graphic structure of ANP, we can say, this method is to correct unstable values of arcs by the stable ones of arcs.

First I explain the concept of "the dominant alternatives" through the model like example 3 (in section 2). To make our discussion systematic, we denote the criteria $C_{1}, C_{2}$ and alternatives by $A_{1}, A_{2}, A_{3}$, then we have ANP problem shown in Fig. 4. The supermatrix is the same as that of 


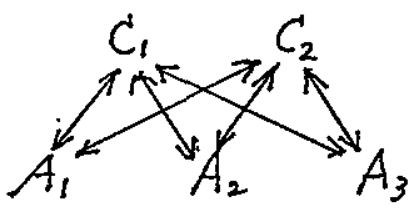

Fig. 4.

(2.4).

Generally the elements of $W$ (the evaluation matrix of criteria by alternatives) are less stable than that of $\boldsymbol{U}$ (the evaluation matrix of alternatives by criteria). The idea of $\mathrm{K} \& \mathrm{~N}$ is to correct the values of $\boldsymbol{W}$ by that of $\boldsymbol{U}$.

The first step is to select a dominant alternative as a representative, say $A_{1}$, and estimate the evaluation values $w_{i j}(j \neq i)$ of other alternatives based on the evaluation value $w_{i 1}$ of the dominant alternative.

The estimation is based on the following ratio or proportion principle;

$$
\frac{w_{i j}}{w_{i 1}}=\frac{u_{j i}}{u_{1 i}}
$$

which says that the ratio of the evaluation of criterion $C_{i}$ by alternative $A_{j}$ to that by $A_{1}$ is equal to the ratio of evaluation of $A_{j}$ by $C_{i}$ to that of $A_{1}$ by $C_{i}$.

The validity of this principle is of course a problem. But if we agree with it, then we can estimate $w_{i j}$ by $u_{j i}, u_{1 i}$ and $w_{i 1}$. Denoting the estimate by $\widehat{w}_{i j}(1)$ we have

$$
\widehat{w}_{i j}(1)=\frac{u_{j i}}{u_{1 i}} w_{i 1}(i=1,2, j=2,3)
$$

Of course the original data $w_{i j}$ does not coincide with $\widehat{w}_{i j}(1)$, and coordinating procedure the original data and the estimation is the "concurrent convergent method".

Generally if we select $\boldsymbol{A}_{\boldsymbol{k}}$ as a dominant alternative, then we have the estimations based on $\boldsymbol{A}_{\boldsymbol{k}}$ like

$$
\widehat{w}_{i j}(k)=\frac{u_{j i}}{u_{k i}} w_{i k}(k=1,2,3, i=1,2, j(\neq k)=1,2,3)
$$

The concurrent convergent procedures are made by calculating the average $w_{i j}^{\prime}$ of original data $w_{i j}$ and estimated $\widehat{w}_{i j}(k)$, and taking this average value $w_{i j}^{\prime}$ as a new original data. These are shown in Table 3. Here $\widehat{\hat{w}}_{i j}(k)$ is a standardized value of $\widehat{w}_{i j}(k)$ in $A_{j}$, that is

$$
\widehat{\hat{w}}_{i j}(k)=\widehat{w}_{i j}(k) /\left(\widehat{w}_{1 j}(k)+\widehat{w}_{2 j}(k)\right)
$$

\section{Example 5}

The concurrent convergent procedure for the following example is shown in Table 4.

$$
U=\left[\begin{array}{ll}
u_{11} & u_{12} \\
u_{21} & u_{22} \\
u_{31} & u_{32}
\end{array}\right]=\left[\begin{array}{ll}
1 / 6 & 0.6 \\
1 / 3 & 0.3 \\
1 / 2 & 0.1
\end{array}\right]
$$


Table 3.

\begin{tabular}{|cc|cc|cc|}
\multicolumn{1}{c}{$A_{1}$} & \multicolumn{2}{c}{$A_{2}$} & \multicolumn{2}{c|}{$A_{3}$} \\
\hline$w_{11}$ & $w_{21}$ & $w_{12}$ & $w_{22}$ & $w_{13}$ & $w_{23}$ \\
& & $\hat{\dot{w}}_{12}(1)$ & $\widehat{\hat{w}}_{22}(1)$ & $\widehat{\hat{w}}_{13}(1)$ & $\widehat{\hat{w}}_{23}(1)$ \\
$\widehat{\hat{w}}_{11}(2)$ & $\widehat{\dot{w}}_{21}(2)$ & & & $\widehat{\hat{w}}_{13}(2)$ & $\widehat{\hat{w}}_{23}(2)$ \\
$\widehat{\hat{w}}_{11}(3)$ & $\widehat{\hat{w}}_{21}(3)$ & $\widehat{\hat{w}}_{12}(3)$ & $\widehat{\dot{w}}_{22}(3)$ & & \\
\hline$w_{11}^{\prime}$ & $w_{21}^{\prime}$ & $w_{12}^{\prime}$ & $w_{22}^{\prime}$ & $w_{13}^{\prime}$ & $w_{23}^{\prime}$ \\
$\vdots$ & $\vdots$ & $\vdots$ & $\vdots$ & $\vdots$ & $\vdots$ \\
\hline
\end{tabular}

$$
W=\left[\begin{array}{lll}
w_{11} & w_{12} & w_{13} \\
w_{21} & w_{22} & w_{33}
\end{array}\right]=\left[\begin{array}{lll}
0.4 & 0.7 & 0.2 \\
0.6 & 0.3 & 0.8
\end{array}\right]
$$

Table 4.

\begin{tabular}{|l|cc|cc|cc|}
\hline step1 & .400 & .600 & .700 & .300 & .200 & .800 \\
& & & .727 & .273 & .923 & .077 \\
& .368 & .632 & & & .913 & .087 \\
& .014 & .986 & .053 & .947 & & \\
\hline step2 & .261 & .739 & .493 & .507 & .679 & .321 \\
& & & .585 & .415 & .864 & .136 \\
& .196 & .804 & & & .814 & .186 \\
& .105 & .895 & .319 & .681 & & \\
\hline step3 & .187 & .813 & .466 & .534 & .786 & .214 \\
& & & .479 & .521 & .806 & .194 \\
& .179 & .821 & & & .797 & .203 \\
& .169 & .831 & .449 & .551 & & \\
\hline step4 & .178 & .822 & .465 & .535 & .796 & .204 \\
& $\overline{w_{11}}$ & $\overline{w_{21}}$ & $\overline{w_{12}}$ & $\overline{w_{22}}$ & $\overline{w_{13}}$ & $\overline{w_{23}}$ \\
\hline
\end{tabular}

Thus we have estimated $\bar{W}$

$$
\bar{W}=\left[\begin{array}{lll}
0.178 & 0.465 & 0.796 \\
0.822 & 0.535 & 0.204
\end{array}\right]
$$

\section{Strongly regular design in AHP}

The last topic is, statistically speaking, a design problem. It is often said that we need a large number ${ }_{n} C_{2}=n(n-1) / 2$ of paired comparisons in AHP if the number $n$ of objects is large. But, I think, we have no difficulties for this if we use well designed sampling method. Roughly speaking, about two times $(2 n)$ of the number $(n)$ of objects are enough for the number of comparisons.

Considering an (undirected) edge $(i, j)$ for paired comparison of object $i$ and $j$, we have a graph for a design. So a design is represented by an (undirected) graph. Wang \& Takahashi[Wang,1998] proposed designs by strongly regular graphs, and showed the goodness of them for various values of $n$ (the number of objects) and $m^{\prime}$ (the number of comparisons).

The definition and properties of a strongly regular graph are well represented by its adjacent matrix, which is a $n \times n$ matrix $N=\left[c_{i j}\right]$ with 0,1 elements; $c_{i j}=1$ if there is an edge $(i, j)$ and $c_{i j}=0$ 
other wise.

The regular graph is well known. Every point of this graph has the same number (d) of edges incident with it, which is represented by

$$
N J=d J(J \text { is all } 1 \text { matrix })
$$

A regular graph is strongly regular (s.r.) if and only if its adjacent matrix $N$ satisfies the following relation;

$$
N^{2}=a_{0} I+a_{1} N+a_{2} J
$$

with integers $a_{0}, a_{1}, a_{2}$, which means that the algebra generated by $N$ and $J$ is linear, that is, any

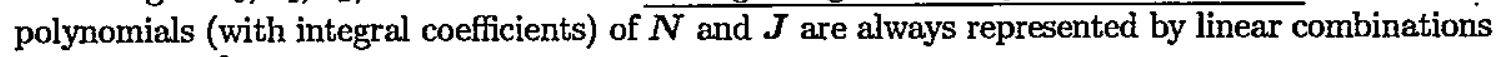
of $N, I\left(=N^{0}\right)$ and $J$.

The strongly regular graph has very nice properties for design. For fairly large domain of combinations of $n$ and $m$, strongly regular designs, if they exist, have smaller standard errors of estimation of weights than any other designs. We can infer that the goodness of strongly regularity is based on its algebraic simplicity shown in (5.2).

But unfortunately we have strongly regular graphs for only very rare combinations of $n$ and $m$. So we extend the concept of s.r to quasi strongly regular (q.s.r). If the adjacent matrix $N$ of regular graph has the following relation for fairly small $r(<<n)$ we call it q.s.r.

$$
N^{r+1}=a_{0} I+a_{1} N+\cdots+a_{r} N^{r}+a_{r+1} J
$$

The q.s.r. graphs can cover the fairly large scope of $n$ and $m$.

W \& $\mathrm{T}$ surveyed various construction methods of s.r. and q.s.r. graphs[Wang,1998]. We can use them for practical implementation in ANP.

\section{References}

[Saaty,1980] Saaty, T. L. (1980) The Analytic Hierarchy Process, McGrawHill, NewYork.

[Saaty,1996] Saaty, T. L. (1996) The Analytic Network Process, RWS Publications.

[Sekitani,1999] Sekitani, K and Yamaki, N (1999) A Logical Interpretation for the Eigenvalue Method in AHP, Journal of Operations Research Society of Japan, Vol. 42, No. 2.

[Sekitani,ISAHP99] Sekitani, K and Yamaki, N (1999) Model based AHP, ISAHP'99 proceedings.

[Sekitani] Sekitani, K and Takahashi, I (submitted) A unified model and analysis for AHP and ANP, Journal of Operations Research Society of Japan.

[Kinoshita, 1999] Kinoshita, E and Nakanishi, M (1999) Proposal of New AHP Model in Light of Dominant Relationship Among Alternatives, Journal of Operations Research Society of Japan, Vol. 42, No. 2.

[Wang,1998] Wang, K and Takahashi, I (1998) How to select paired comparisons in AHP of incomplete information - Strongly regular graph design, Journal of Operations Research Society of Japan, Vol. 41, No. 2, 311-328. 